\title{
On the Origin of the Harmonic Term in Noncommutative Quantum Field Theory ${ }^{\star}$
}

Axel de GOURSAC

Département de Mathématiques, Université Catholique de Louvain, Chemin du Cyclotron, 2, 1348 Louvain-la-Neuve, Belgium

E-mail: axelmg@melix.net

Received March 30, 2010, in final form June 01, 2010; Published online June 09, 2010

doi:10.3842/SIGMA.2010.048

\begin{abstract}
The harmonic term in the scalar field theory on the Moyal space removes the UV-IR mixing, so that the theory is renormalizable to all orders. In this paper, we review the three principal interpretations of this harmonic term: the Langmann-Szabo duality, the superalgebraic approach and the noncommutative scalar curvature interpretation. Then, we show some deep relationship between these interpretations.
\end{abstract}

Key words: noncommutative QFT; gauge theory; renormalization; Heisenberg algebra

2010 Mathematics Subject Classification: 81T13; 81T15; 81T75

\section{Introduction}

Since around fifteen years, the interest for noncommutative geometry has been growing in physics. In many domains of mathematics, like topology, measure theory, geometry, one has noticed that spaces are equivalently described by the commutative algebras of their functions. Then, noncommutative geometry [1,2] considers noncommutative algebras as corresponding to some "noncommutative spaces". A particular type of noncommutative algebras is given by deformation quantization of symplectic spaces. This is the case of the Moyal space [3], and it can be physically interpreted as the fact that one cannot know simultaneously positions in the space with an arbitrary precision, but at a certain scale (the Planck scale) events are no more localizable [4]. Physical theories on such noncommutative spaces, for instance noncommutative quantum field theory, are then candidates for new physics beyond the Standard Model of particle physics. Of course, the study of gauge theory on noncommutative spaces is of fundamental importance, as it is the case in the Standard Model.

On the Euclidean Moyal space, the real scalar $\phi^{4}$ theory is not renormalizable, because of a new type of divergence called the Ultraviolet-Infrared (UV/IR) mixing [5], and which seems to be generic on several noncommutative spaces $[6,7]$. Renormalizability is however a fundamental property for physical field theories. Recently, a solution has been proposed [8] by adding a harmonic term in the action, so that the theory is renormalizable at all orders. As we will see it in this paper, this model has new interesting flow properties, which do not appear in the usual commutative scalar theory. The mathematical understanding of this harmonic term is of first importance since it may permit to generalize this solution to other noncommutative spaces.

We propose here to review the three principal mathematical interpretations of the harmonic term: the Langmann-Szabo duality [9] and its group formulation (in [10]), the superalgebraic approach [11], and the interpretation in terms of a noncommutative scalar curvature [12, 13].

\footnotetext{
*This paper is a contribution to the Special Issue "Noncommutative Spaces and Fields". The full collection is available at http://www.emis.de/journals/SIGMA/noncommutative.html
} 
Then, we show how the Langmann-Szabo duality can be reformulated in the superalgebraic framework at the group level, and we underline the relationship between the three viewpoints.

The paper is organized as follows. We first present the Moyal space and the UV/IR mixing in Subsections 2.1 and 2.2. Then, the solution with harmonic term is exposed with its properties in Subsection 2.3, so as its associated gauge theory, obtained by an effective action, in Subsection 2.4. The three already quoted possible origins of the harmonic term are reviewed in Section 3. Finally, we discuss the unification and the relations between these interpretations in Section 4.

\section{Quantum field theory on the Moyal space}

\subsection{Presentation of the Moyal space}

We expose in this subsection the basic definitions and properties of the Moyal space [3] for the paper to be self-contained. The Moyal space is a deformation quantization of the space $\mathbb{R}^{D}$, for an even dimension $D$. Let $\Sigma$ be the symplectic form on $\mathbb{R}^{D}$ represented in the canonical basis by the matrix:

$$
\Sigma=\left(\begin{array}{ccccc}
0 & -1 & 0 & 0 & \\
1 & 0 & 0 & 0 & \\
0 & 0 & 0 & -1 & \ddots \\
0 & 0 & 1 & 0 & \\
& & \ddots & &
\end{array}\right),
$$

and let $\Theta=\theta \Sigma$, where $\theta$ is a real parameter of the deformation. On $\mathcal{S}\left(\mathbb{R}^{D}\right)$, the $\mathbb{C}$-valued Schwartz functions space on $\mathbb{R}^{D}$, is defined the following associative noncommutative product: $\forall f, g \in \mathcal{S}\left(\mathbb{R}^{D}\right), \forall x \in \mathbb{R}^{D}$,

$$
(f \star g)(x)=\frac{1}{\pi^{D} \theta^{D}} \int \mathrm{d}^{D} y \mathrm{~d}^{D} z f(x+y) g(x+z) e^{-i y \wedge z},
$$

where $y \wedge z=2 y_{\mu} \Theta_{\mu \nu}^{-1} z_{\nu}$ (we use in this paper the Einstein summation convention). It is called the Moyal product, and turns $\mathcal{S}\left(\mathbb{R}^{D}\right)$ into a topological $*$-algebra (with the usual involution ${ }^{\dagger}$ ).

Then, this product can be extended on the temperated distributions $\mathcal{S}^{\prime}\left(\mathbb{R}^{D}\right)$ by duality, and one consider $\mathcal{M}_{\theta}$, the algebra of (left and right) multipliers of $\mathcal{S}\left(\mathbb{R}^{D}\right)$ in $\mathcal{S}^{\prime}\left(\mathbb{R}^{D}\right)$, endowed with the Moyal product. $\mathcal{M}_{\theta}$ is also a topological *-algebra involving $\mathcal{S}\left(\mathbb{R}^{D}\right)$ and polynomial functions for instance. See $[14,15]$ for more details.

The limit $\theta \rightarrow 0$ is called the commutative limit because the Moyal product

$$
\left.(f \star g)(x)\right|_{\theta=0}=f(x) g(x)
$$

is the usual pointwise commutative product in this limit. Moreover, the integral is a trace for the Moyal product since if $f, g \in \mathcal{M}_{\theta}$ such that $f \star g \in L^{1}\left(\mathbb{R}^{D}\right)$, one has

$$
\int \mathrm{d}^{D} x(f \star g)(x)=\int \mathrm{d}^{D} x f(x) g(x) .
$$

The usual derivatives $\partial_{\mu}$ are inner derivations:

$$
\partial_{\mu} f=-\frac{i}{2}\left[\widetilde{x}_{\mu}, f\right]_{\star},
$$


where $\widetilde{x}_{\mu}=2 \Theta_{\mu \nu}^{-1} x_{\nu}$, the commutator is $[f, g]_{\star}=f \star g-g \star f$ and the anticommutator $\{f, g\}_{\star}=$ $f \star g+g \star f$. Note that the coordinate functions satisfy the following commutation relation:

$$
\left[x_{\mu}, x_{\nu}\right]_{\star}=i \Theta_{\mu \nu} .
$$

Finally, the Moyal space is a spectral triple of the non-compact type [16], and its spectral distance has been computed in [17].

\subsection{The UV-IR mixing}

The straightforward generalization of the real $\phi^{4}$ theory on the Euclidean Moyal space:

$$
S(\phi)=\int \mathrm{d}^{D} x\left(\frac{1}{2} \partial_{\mu} \phi \star \partial_{\mu} \phi+\frac{m^{2}}{2} \phi \star \phi+\lambda \phi \star \phi \star \phi \star \phi\right),
$$

suffers from a new type of divergence, called the ultraviolet-infrared (UV/IR) mixing [5]. Let us here analyze this divergence. Thanks to property (2), the action is given by:

$$
S(\phi)=\int \mathrm{d}^{D} x\left(\frac{1}{2}\left(\partial_{\mu} \phi\right)^{2}+\frac{m^{2}}{2} \phi^{2}+\lambda \phi \star \phi \star \phi \star \phi\right),
$$

so that only the interaction is changed by a non-local term. Any vertex of this theory admits only cyclic permutations as symmetries and not all permutations like in the commutative case (for $\theta=0$ ). In the Fourier space, the Feynman rules are:

- $\frac{1}{p^{2}+m^{2}}$ for a propagator,

- $\lambda e^{i \frac{\theta^{2}}{4}\left(p_{1} \wedge p_{2}+p_{1} \wedge p_{3}+p_{2} \wedge p_{3}\right)}$ for a vertex,

where $\left(p_{1}, p_{2}, p_{3}, p_{4}\right)$ are the incoming impulsions to the vertex, with respect to its cyclic order.

By taking into account the cyclic order of each vertex, the Feynman graphs can be divided into two sectors: planar graphs and non-planar ones (for more details, see [18]). The nonplanar graphs have new IR-divergences, called UV/IR mixing. Let us see such an example of divergence, which is typical of the UV/IR mixing as shown in [19]. The computation of the amplitude associated to the non-planar tadpole (see Fig. 1) gives rise to

$$
\mathcal{A}(p)=\frac{\lambda}{(2 \pi)^{D}} \int \mathrm{d}^{D} k \frac{e^{\frac{i \theta^{2}}{2} k \wedge p}}{k^{2}+m^{2}}=\frac{\lambda}{(2 \pi)^{\frac{D}{2}}}\left(\frac{m^{2}}{\theta^{2} p^{2}}\right)^{\frac{D-2}{4}} K_{\frac{D}{2}-1}(m \theta|p|),
$$

where $p$ is the external impulsion and $K$ is a modified Bessel function. This amplitude is finite for a fixed external impulsion $p \neq 0$, but singular by taking the limit $|p| \rightarrow 0$. Indeed, in $D=4$ dimensions, $\mathcal{A}(p) \propto_{p \rightarrow 0} \frac{1}{p^{2}}$.

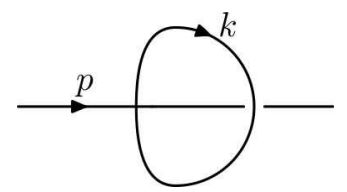

Figure 1. Non-planar tadpole.

Then, if this non-planar tadpole diagram is inserted into higher-loop order graphs, this impulsion $p$ is integrated over and can produce an IR-divergence. Since this divergence comes from the noncommutativity of the limit $\Lambda \rightarrow \infty$, for an UV regularization $\Lambda$ of the variable $k$, and of the IR-limit $|p| \rightarrow 0$, it is called UV/IR mixing. And this divergence cannot be renormalized by counterterms of the form of the initial action (3), so that the theory is not renormalizable. Numerical evidence for the effects induced by the UV/IR mixing can be found for example in $[20,21]$. 


\subsection{Noncommutative scalar field theory with harmonic term}

The first solution to this problem of UV/IR mixing in the real scalar field theory has been proposed by H. Grosse and R. Wulkenhaar [8] by adding a harmonic term in the action:

$$
S(\phi)=\int \mathrm{d}^{D} x\left(\frac{1}{2}\left(\partial_{\mu} \phi\right)^{2}+\frac{\Omega^{2}}{2} \widetilde{x}^{2} \phi^{2}+\frac{m^{2}}{2} \phi^{2}+\lambda \phi \star \phi \star \phi \star \phi\right),
$$

where $\Omega$ is a real parameter. The propagator of this theory is changed and given by the Mehler kernel in the position space [22]:

$$
\begin{aligned}
& C(x, y)=\frac{\theta}{4 \Omega}\left(\frac{\Omega}{\pi \theta}\right)^{\frac{D}{2}} \int_{0}^{\infty} \frac{\mathrm{d} \alpha}{\sinh ^{\frac{D}{2}}(\alpha)} e^{-\frac{m^{2} \alpha}{2 \Omega}} C(x, y, \alpha), \\
& C(x, y, \alpha)=\exp \left(-\frac{\widetilde{\Omega}}{4} \operatorname{coth}\left(\frac{\alpha}{2}\right)(x-y)^{2}-\frac{\widetilde{\Omega}}{4} \tanh \left(\frac{\alpha}{2}\right)(x+y)^{2}\right) .
\end{aligned}
$$

where $\widetilde{\Omega}=\frac{2 \Omega}{\theta}$.

Of course, this theory breaks now the translation invariance (indeed $C(x, y) \neq C(x-y)$ ). But this lack of translation invariance is responsible of the removing of the UV/IR mixing. The amplitude of the non-planar tadpole (Fig. 1) has the same behavior when $|p| \rightarrow 0$ as before (see Subsection 2.2), but the propagators joining these non-planar tadpoles in a higher loop order graph are now of the type (5) and remove the IR-divergence thanks to this translational symmetry breaking [23]. And the theory (4) has been shown to be renormalizable to all orders in perturbation in $D=4[8,24,25,26]$ and superrenormalizable in $D=2$ [27]. A parametric representation of this model has been provided in [28, 29] and a Connes-Kreimer Hopf algebra encoding its renormalization has also been constructed in [30, 31].

Let us study some properties of the noncommutative scalar field theory with harmonic term (4).

- Concerning the renormalization flows, it has been shown up to three loops that the constant $\Omega$ was running towards a fixed point $\Omega=1$ [32]. At this fixed point, the beta function of the coupling constant $\lambda$ vanishes up to irrelevant terms at all orders of perturbation [33, 34], so that the theory (4) does not involve any Landau ghost, contrary to the commutative $\phi^{4}$ model.

- Moreover, the vacuum solutions of the theory which respect the same symmetry have been exhibited in every dimensions $[35,36]$, in view of a possible spontaneous symmetry breaking.

- Furthermore, even if the deformation quantization, and in particular the choice of the symplectic structure $\Sigma$ with $\Theta=\theta \Sigma$, breaks the rotation group symmetry, it has been shown [37] that the rotational invariance is fully restored at the classical and quantum level (at all orders in perturbation) by considering a family of actions labeled by the symplectic structures of a certain orbit of the rotation group.

Note that there are now other renormalizable theories on the Moyal space. For instance, the LSZ-model [38] and the Gross-Neveu model [39], respectively in the complex scalar case $(D=4)$ and in the fermionic case $(D=2)$. Another renormalizable real scalar model on the Moyal space has been exhibited [40], in which the non-local IR counterterm $\frac{1}{p^{2}} \phi^{2}$ is now included in the classical action. The resulting theory is translation-invariant, but does not possess the properties exposed above for the Grosse-Wulkenhaar model (see also [41, 42, 19, 43, 44]). 


\subsection{The associated gauge theory}

Let us first introduce the noncommutative framework adapted to the $U(1)$-gauge theory on the Moyal space. See $[45,46]$ for more details. Gauge potentials are real elements $A_{\mu} \in \mathcal{M}_{\theta}$ $\left(A_{\mu}^{\dagger}=A_{\mu}\right)$ and the associated covariant derivatives can be expressed as $\forall \phi \in \mathcal{M}_{\theta}$,

$$
\nabla_{\mu} \phi=\partial_{\mu} \phi-i A_{\mu} \star \phi .
$$

In this setting, gauge transformations are determined by unitary elements $g \in \mathcal{M}_{\theta}\left(g^{\dagger} \star g=\right.$ $g \star g^{\dagger}=1$ ), and act on the fields as

$$
\phi^{g}=g \star \phi, \quad A_{\mu}^{g}=g \star A_{\mu} \star g^{\dagger}+i g \star \partial_{\mu} g^{\dagger},
$$

so that $\nabla_{\mu} \phi$ transforms as: $\left(\nabla_{\mu} \phi\right)^{g}=g \star\left(\nabla_{\mu} \phi\right)$. Note that the gauge theory has also an adjoint action on complex scalar fields $\varphi \mapsto g \star \varphi \star g^{\dagger}$, as it will be the case in Subsection 3.2. Like in the commutative case (but for non-Abelian theories), the curvature of the potential $A_{\mu}$ takes the form:

$$
F_{\mu \nu}=\partial_{\mu} A_{\nu}-\partial_{\nu} A_{\mu}-i\left[A_{\mu}, A_{\nu}\right]_{\star},
$$

and transforms covariantly under gauge transformations: $F_{\mu \nu}^{g}=g \star F_{\mu \nu} \star g^{\dagger}$.

A major difference with the commutative case is the existence of a canonical gauge-invariant connection. By setting $\xi_{\mu}=-\frac{1}{2} \widetilde{x}_{\mu}$, it turns out that the gauge potential $A_{\mu}^{\text {inv }}=\xi_{\mu}$ defines a connection invariant under gauge transformations: $\left(A_{\mu}^{\mathrm{inv}}\right)^{g}=g \star A_{\mu}^{\mathrm{inv}} \star g^{\dagger}+i g \star \partial_{\mu} g^{\dagger}=A_{\mu}^{\mathrm{inv}}$. Let us introduce the covariant coordinates $\mathcal{A}_{\mu}=A_{\mu}-A_{\mu}^{\text {inv }}$, transforming also covariantly: $\mathcal{A}_{\mu}^{g}=g \star \mathcal{A}_{\mu} \star g^{\dagger}$.

The natural action for the $U(1)$-gauge theory on the Moyal space is

$$
S(A)=\int \mathrm{d}^{D} x\left(\frac{1}{4} F_{\mu \nu} \star F_{\mu \nu}\right),
$$

but it suffers also from the UV/IR mixing, which renders its renormalizability quite unlikely [47]. Indeed, by taking into account the ghost contribution, the "non-planar" polarization tensor is finite for a fixed external impulsion $p \neq 0$, but singular in $D=4$ by taking the limit $|p| \rightarrow 0$ :

$$
\Pi_{\mu \nu}^{\mathrm{np}}(p) \propto_{p \rightarrow 0} \frac{\widetilde{p}_{\mu} \widetilde{p}_{\nu}}{p^{4}} .
$$

The same phenomenon as in the scalar theory appears (see Subsection 2.2), and one can hope that an analogous solution of the problem of UV/IR mixing can be found.

By investigating what could be the analogue of the harmonic term for gauge theory, a gauge action has been computed from the Grosse-Wulkenhaar model coupled with gauge fields by a one-loop effective action $[45,48]$ :

$$
S(A)=\int \mathrm{d}^{D} x\left(\frac{1}{4} F_{\mu \nu} \star F_{\mu \nu}+\frac{\Omega^{2}}{4}\left\{\mathcal{A}_{\mu}, \mathcal{A}_{\nu}\right\}_{\star}^{2}+\kappa \mathcal{A}_{\mu} \star \mathcal{A}_{\mu}\right),
$$

where $\Omega$ and $\kappa$ are new real parameters. This gauge-invariant action is naturally associated to the scalar theory with harmonic term (4) and is therefore a good candidate to renormalizability. Note that the two additional terms are typically noncommutative since they depend on $\mathcal{A}_{\mu}$ and on the existence of a canonical connection.

Upon expanding the quadratic term in $A_{\mu}$ of the action (7), by using $\mathcal{A}_{\mu}=A_{\mu}+\frac{1}{2} \widetilde{x}_{\mu}$, one finds:

$$
\int \mathrm{d}^{D} x\left(-\frac{1}{2} A_{\mu} \partial^{2} A_{\mu}+\frac{\Omega^{2}}{2} \widetilde{x}^{2} A_{\mu} A_{\mu}+\kappa A_{\mu} A_{\mu}-\frac{1}{2}\left(1-\Omega^{2}\right)\left(\partial_{\mu} A_{\mu}\right)^{2}+\Omega^{2}\left(\widetilde{x}_{\mu} A_{\mu}\right)^{2}\right) .
$$


Except the last two (off-diagonal) terms, which need to be suppressed by an appropriate gauge fixing, this expression is exactly the quadratic operator of the scalar theory (4) applied on the field $A_{\mu}$, with a harmonic term and a mass term, and whose inverse is the Mehler kernel, responsible of the removing of the UV/IR mixing in the scalar case. Notice that the ghost sector of a similar gauge model has been studied in $[49,50]$.

However, the action involves also a linear part in $A_{\mu}$ :

$$
\int \mathrm{d}^{D} x\left(\frac{\Omega^{2}}{2} \widetilde{x}^{2} \widetilde{x}_{\mu} A_{\mu}+\kappa \widetilde{x}_{\mu} A_{\mu}\right)
$$

which means that $A_{\mu}=0$ is not a solution of the equation of motion. A simple vacuum $A_{\mu}=A_{\mu}^{\text {inv }}$ (or equivalently $\mathcal{A}_{\mu}=0$ ) leads to non-dynamical matrix model [51]. The other vacuum solutions have been exhibited in [52]. For a review on noncommutative QED with strong background fields, see [53].

\section{The possible origins of the harmonic term}

In this section, we review the different mathematical interpretations of the harmonic term of the model (4), which is responsible of the renormalizability and of the special flow properties of this noncommutative theory.

\subsection{Langmann-Szabo duality}

The Grosse-Wulkenhaar model (4) has a special symmetry pointed out by Langmann and Szabo [9], and called the Langmann-Szabo duality. This symmetry is in fact a (cyclic) symplectic Fourier transformation: for $\phi$ a real scalar field,

$$
\hat{\phi}\left(k_{a}\right)=\frac{1}{(\pi \theta)^{\frac{D}{2}}} \int \mathrm{d}^{D} x \phi(x) e^{-i(-1)^{a} k_{a} \wedge x}
$$

where $a \in\{1, \ldots, 4\}$ denotes the position of $\hat{\phi}\left(k_{a}\right)$ in a product $\hat{\phi} \star \cdots \star \hat{\phi}$, or equivalently the (cyclic) order of the external impulsion $k_{a}$ in a vertex. This Fourier transformation differs from the usual one $\mathcal{F}$ only by the change of variable $k \rightarrow \pm \widetilde{k}$.

It is then possible to show the following properties:

$$
\begin{aligned}
& \int \mathrm{d}^{D} x \phi^{2}(x)=\int \mathrm{d}^{D} k(\hat{\phi} \star \hat{\phi})(k), \\
& \int \mathrm{d}^{D} x(\phi \star \phi \star \phi \star \phi)(x)=\int \mathrm{d}^{D} k(\hat{\phi} \star \hat{\phi} \star \hat{\phi} \star \hat{\phi})(k), \\
& \widehat{\partial_{\mu} \phi}\left(k_{a}\right)=-i(-1)^{a}\left(\widetilde{k}_{a}\right)_{\mu} \hat{\phi}\left(k_{a}\right), \quad \widehat{\left(\widetilde{x}_{\mu} \phi\right)}\left(k_{a}\right)=i(-1)^{a} \partial_{\mu}^{k} \hat{\phi}\left(k_{a}\right) .
\end{aligned}
$$

Note that there is a difference of sign in comparison with the usual Parseval-Plancherel equality $\int \mathrm{d}^{D} x \phi^{2}(x)=\int \mathrm{d}^{D} k \mathcal{F}(\phi)(-k) \mathcal{F}(\phi)(k)$ due to the cyclic convention of sign in (9). Upon using these identities, one finds that

$$
\int \mathrm{d}^{D} x\left(\frac{1}{2}\left(\partial_{\mu}^{x} \phi\right)^{2}+\frac{\Omega^{2}}{2} \widetilde{x}^{2} \phi^{2}+\frac{m^{2}}{2} \phi^{2}\right)=\int \mathrm{d}^{D} k\left(\frac{1}{2} \widetilde{k}^{2} \hat{\phi}^{2}+\frac{\Omega^{2}}{2}\left(\partial_{\mu}^{k} \hat{\phi}\right)^{2}+\frac{m^{2}}{2} \hat{\phi}^{2}\right),
$$

so that the action (4) satisfies

$$
S[\phi ; m, \lambda, \Omega]=\Omega^{2} S\left[\hat{\phi}, \frac{m}{\Omega}, \frac{\lambda}{\Omega^{2}}, \frac{1}{\Omega}\right],
$$


called the Langmann-Szabo covariance. At the special point $\Omega=1$, the action is invariant under this duality. This symmetry, which does not apply in the case of (3) without the harmonic term, seems to play a crucial role in spoiling the UV/IR mixing. For an adaptation of this duality to the Minkowskian framework, see [54].

However, the Langmann-Szabo duality is not compatible with gauge symmetry, and more generally not well adapted for actions with cubic terms in the fields. The gauge theory (7) is indeed not covariant under this duality, and it is one major problem of this interpretation of the harmonic term.

In [10], the Langmann-Szabo duality and the quadratic operator $\left(-\partial^{2}+\Omega^{2} \widetilde{x}^{2}\right)$ involved in (4) have been reinterpreted in terms of the classical metaplectic representation constructed from the Heisenberg group. Let us describe this representation, but in different conventions of those used in $[10,55]$, for the link with the superalgebraic interpretation (see Subsection 4.1).

Firstly, consider the phase space $\mathbb{R}^{2 D}$, whose coordinates are positions $x_{\mu}$ and impulsions $p_{\mu}$, and denote by $\omega$ the symplectic structure on $\mathbb{R}^{2 D}$ defined by: for $(x, p),(y, q) \in \mathbb{R}^{2 D}$,

$$
\omega((x, p),(y, q))=x_{\mu} \Sigma_{\mu \nu} q_{\nu}+p_{\mu} \Sigma_{\mu \nu} y_{\nu}
$$

so that the matrix representing $\omega$ is $\left(\begin{array}{cc}0 & \Sigma \\ \Sigma & 0\end{array}\right)$, where $\Sigma$ is given by (1). The Heisenberg algebra is the $\mathbb{R}$-Lie algebra $\mathfrak{h}_{D}=\mathbb{R}^{2 D} \oplus \mathbb{R}$, with the following relations: $\forall(x, p, s),(y, q, t) \in \mathfrak{h}_{D}$,

$$
[(x, p, s),(y, q, t)]=(0,0, \omega((x, p),(y, q))) .
$$

The Heisenberg group $H_{D}$ is homeomorphic to $\mathfrak{h}_{D}$, and the exponential map exp : $\mathfrak{h}_{D} \rightarrow H_{D}$ is the identity in this identification. Then, the group law of $H_{D}$ is given by:

$$
(x, p, s) \cdot(y, q, t)=\left(x+y, p+q, s+t+\frac{1}{2} \omega((x, p),(y, q))\right),
$$

due to the Baker-Campbell-Hausdorff formula. By the Stone-von Neumann theorem, $H_{D}$ admits one unique irreducible unitary representation $\rho$ (up to equivalence), such that $\rho(0,0, s)=$ $e^{-i s} \mathbb{1}$. $\rho$ is called the Schrödinger representation.

Let us introduce the operators $X_{\mu}$ and $P_{\mu}$ on Schwartz functions $f \in \mathcal{S}\left(\mathbb{R}^{D}\right): \forall y \in \mathbb{R}^{D}$,

$$
\left(X_{\mu} f\right)(y)=y_{\mu} f(y), \quad\left(P_{\mu} f\right)(y)=i \Sigma_{\mu \nu} \frac{\partial f}{\partial y_{\nu}}(y)
$$

satisfying: $\left[X_{\mu}, P_{\nu}\right]=i \Sigma_{\mu \nu}$. At the level of the Lie algebra $\mathfrak{h}_{D}$, the infinitesimal representation $\mathrm{d} \rho: \mathfrak{h}_{D} \rightarrow \mathcal{B}\left(\mathcal{S}\left(\mathbb{R}^{D}\right)\right)$ takes the usual form: $\forall(x, p, s) \in \mathfrak{h}_{D}, \forall f \in \mathcal{S}\left(\mathbb{R}^{D}\right), \forall y \in \mathbb{R}^{D}$,

$$
(\mathrm{d} \rho(x, p, s) f)(y)=-i\left(x_{\mu} X_{\mu}+p_{\mu} P_{\mu}+s\right) f(y) .
$$

By exponentiation, one obtains the expression of $\rho$, representation of $H_{D}$ on the Hilbert space $L^{2}\left(\mathbb{R}^{D}\right)$ :

$$
\rho(x, p, s) f(y)=e^{\frac{i}{2} x \Sigma p-i x y-i s} f(y-\Sigma p) .
$$

The symplectic group $S p\left(\mathbb{R}^{2 D}, \omega\right)$, defined by automorphisms $M$ of $\mathbb{R}^{2 D}$ satisfying $M^{T} \omega M=$ $\omega$ in matrix notations, acts naturally on the Heisenberg algebra. A matrix $M=\left(\begin{array}{ll}A & B \\ C & D\end{array}\right)$ belongs to this group if and only if

$$
C^{T} \Sigma A+A^{T} \Sigma C=0, \quad D^{T} \Sigma B+B^{T} \Sigma D=0, \quad D^{T} \Sigma A+B^{T} \Sigma C=\Sigma .
$$


The Lie algebra of this group is denoted by $\mathfrak{s p}\left(\mathbb{R}^{2 D}, \omega\right)$ and contains matrices $\left(\begin{array}{ll}A & B \\ C & D\end{array}\right)$ such that:

$$
(\Sigma A)^{T}=\Sigma D, \quad(\Sigma B)^{T}=\Sigma B, \quad(\Sigma C)^{T}=\Sigma C .
$$

Let us start the construction of the metaplectic representation. If $M \in S p\left(\mathbb{R}^{2 D}, \omega\right)$, consider the operator $\rho \circ M(x, p, s)=\rho(M .(x, p), s)$ for $(x, p, s) \in H_{D} . \rho \circ M$ is a unitary representation of $H_{D}$ on the Hilbert space $L^{2}\left(\mathbb{R}^{D}\right)$ such that $\rho \circ M(0,0, s)=e^{-i s} 1$. Consequently, $\rho \circ M$ and $\rho$ are equivalent: there exists a unitary operator $\mu(M)$ on $L^{2}\left(\mathbb{R}^{D}\right)$ satisfying: $\forall(x, p, s) \in H_{D}$,

$$
\rho \circ M(x, p, s)=\mu(M) \rho(x, p, s) \mu(M)^{-1} .
$$

$\mu: S p\left(\mathbb{R}^{2 D}, \omega\right) \rightarrow \mathcal{L}\left(L^{2}\left(\mathbb{R}^{D}\right)\right)$ is then a unitary projective representation (or a group representation of the double covering of $\left.S p\left(\mathbb{R}^{2 D}, \omega\right)\right)$.

For $w \in \mathbb{R}^{2 D}$ and $N=\left(\begin{array}{cc}A & B \\ C & D\end{array}\right) \in \mathfrak{s p}\left(\mathbb{R}^{2 D}, \omega\right)$, we denote

$$
\begin{aligned}
& \mathbb{P}_{N}(w)=-\frac{1}{2} w N \omega w=-\frac{1}{2} w\left(\begin{array}{cc}
B \Sigma & A \Sigma \\
D \Sigma & C \Sigma
\end{array}\right) w . \\
& \mathbb{P}_{N}(X, P)=-\frac{1}{2} X B \Sigma X-\frac{1}{2} P C \Sigma P-X A \Sigma P+\frac{i}{2} \operatorname{Tr}(A),
\end{aligned}
$$

if $X=\left(X_{\mu}\right)$ and $P=\left(P_{\mu}\right)$ are the $D$-dimensional vectors of the operators on $\mathcal{S}\left(\mathbb{R}^{D}\right)$ defined in (13). Then, the infinitesimal representation $\mathrm{d} \mu: \mathfrak{s p}\left(\mathbb{R}^{2 D}, \omega\right) \rightarrow \mathcal{L}\left(\mathcal{S}\left(\mathbb{R}^{D}\right)\right)$ can be expressed [55] as: $\forall f \in \mathcal{S}\left(\mathbb{R}^{D}\right), \forall y \in \mathbb{R}^{D}$,

$$
(\mathrm{d} \mu(N) f)(y)=-i \mathbb{P}_{N} f(y)=\left(\frac{i}{2} y B \Sigma y-\frac{i}{2} \frac{\partial}{\partial y} \Sigma C \frac{\partial}{\partial y}+y A \frac{\partial}{\partial y}+\frac{1}{2} \operatorname{Tr}(A)\right) f(y) .
$$

Since $\mu\left(e^{N}\right)=e^{\mathrm{d} \mu(N)}$, we deduce the expression of the metaplectic representation (up to a sign): $\forall f \in L^{2}\left(\mathbb{R}^{D}\right)$,

$$
\begin{aligned}
& \text { if } M=\left(\begin{array}{cc}
A & 0 \\
0 & D
\end{array}\right) \in S p\left(\mathbb{R}^{2 D}, \omega\right), \quad \mu(M) f(y)=|\operatorname{det}(A)|^{\frac{1}{2}} f\left(A^{T} y\right), \\
& \text { if } M=\left(\begin{array}{cc}
1 & B \\
0 & \mathbb{1}
\end{array}\right) \in S p\left(\mathbb{R}^{2 D}, \omega\right), \quad \mu(M) f(y)=e^{\frac{i}{2} y B \Sigma y} f(y), \\
& \text { if } M=\left(\begin{array}{cc}
0 & \Sigma \\
\Sigma & 0
\end{array}\right) \in S p\left(\mathbb{R}^{2 D}, \omega\right), \quad \mu(M) f(y)=i^{\frac{D}{2}} \mathcal{F}^{-1} f(y),
\end{aligned}
$$

where the (usual) Fourier transformation is given by

$$
\mathcal{F} f(y)=\frac{1}{(2 \pi)^{\frac{D}{2}}} \int \mathrm{d}^{D} z f(z) e^{i y z}
$$

and the group $S p\left(\mathbb{R}^{2 D}, \omega\right)$ is generated by the above matrices.

Before reformulating the interpretation of the Langmann-Szabo duality with the metaplectic representation, we prove the following classical lemma.

Lemma 1. For $M \in S p\left(\mathbb{R}^{2 D}, \omega\right)$ and $N \in \mathfrak{s p}\left(\mathbb{R}^{2 D}, \omega\right)$, one has:

$$
\mathrm{d} \mu\left(\operatorname{Ad}_{M} N\right)=\mu(M)(\mathrm{d} \mu(N)) \mu(M)^{-1} .
$$


Proof. Indeed,

$$
\mathrm{d} \mu\left(\operatorname{Ad}_{M} N\right)=\frac{\mathrm{d}}{\mathrm{d} t} \mu\left(e^{t \operatorname{Ad}_{M} N}\right)_{\mid t=0}=\frac{\mathrm{d}}{\mathrm{d} t} \mu\left(\operatorname{Ad}_{M} e^{t N}\right)_{\mid t=0}=\mu(M)(\mathrm{d} \mu(N)) \mu(M)^{-1} .
$$

The quadratic operator involved in (4) can be obtained from the infinitesimal metaplectic representation as follows:

$$
\text { if } Z_{\Omega}=\left(\begin{array}{cc}
0 & \frac{4 \Omega^{2}}{\theta^{2}} \Sigma \\
\Sigma & 0
\end{array}\right), \quad \mathrm{d} \mu\left(Z_{\Omega}\right)=-\frac{i}{2}\left(-\partial^{2}+\Omega^{2} \widetilde{y}^{2}\right)
$$

Let us now investigate the elements $M=\left(\begin{array}{ll}A & B \\ C & D\end{array}\right) \in S p\left(\mathbb{R}^{2 D}, \omega\right)$ which leave $Z_{\Omega}$ invariant or covariant.

Definition 1. A matrix $M$ leaves $Z_{\Omega}$ covariant if $\operatorname{Ad}_{M} Z_{\Omega}$ is of the form of $Z_{\Omega}$ itself, namely

$$
\operatorname{Ad}_{M} Z_{\Omega}=\left(\begin{array}{cc}
0 & \alpha \Sigma \\
\beta \Sigma & 0
\end{array}\right)
$$

for $\alpha, \beta \in \mathbb{R}$. This is a reformulation of the Langmann-Szabo covariance (11).

Then, by applying Lemma 1, the operators $\mu(M)$ applied on the field $\phi$ will then leave the quadratic part of the theory (4) invariant or covariant. The elements $M$ of $S p\left(\mathbb{R}^{2 D}, \omega\right)$ leaving $Z_{\Omega}$ covariant have to satisfy:

$$
B D^{T}+\frac{4 \Omega^{2}}{\theta^{2}} A C^{T}=0, \quad B B^{T}+\frac{4 \Omega^{2}}{\theta^{2}} A A^{T}=\alpha \mathbb{1}, \quad D D^{T}+\frac{4 \Omega^{2}}{\theta^{2}} C C^{T}=\beta \mathbb{1} .
$$

Consider now the two opposite typical cases.

- If $B=C=0$, one obtains the group $O\left(\mathbb{R}^{D}, \mathbb{1}\right) \cap S p\left(\mathbb{R}^{D}, \Sigma\right)$, which was already known to let the theory (4) invariant (see [37]).

- If $A=D=0$, for instance $M=M_{\mathrm{LS}}=\frac{\theta}{2}\left(\begin{array}{cc}0 & \frac{4}{\theta^{2}} \mathbb{1} \\ \mathbb{1} & 0\end{array}\right)$, then $\operatorname{Ad}_{M} Z_{\Omega}=\left(\begin{array}{cc}0 & \frac{4}{\theta^{2}} \Sigma \\ \Omega^{2} \Sigma & 0\end{array}\right)$. Since $M_{\mathrm{LS}}=\left(\begin{array}{cc}-\frac{2}{\theta} \Sigma & 0 \\ 0 & -\frac{\theta}{2} \Sigma\end{array}\right) \omega$, one can compute the operator $\mu\left(M_{\mathrm{LS}}\right)$ thanks to equations (14): $\forall f \in L^{2}\left(\mathbb{R}^{D}\right)$,

$$
\mu\left(M_{\mathrm{LS}}\right) f(y)=\frac{i^{\frac{D}{2}}}{(\pi \theta)^{\frac{D}{2}}} \int \mathrm{d}^{D} z f(z) e^{-i y \wedge z}=i^{\frac{D}{2}} \hat{f}(y),
$$

that is the symplectic Fourier transformation (9) (with even $a$ ).

The Langmann-Szabo duality, at the quadratic level of the action (4), corresponds then to the adjoint action of $M_{\mathrm{LS}}$ on $Z_{\Omega}$ (see Lemma 1), and it is a symmetry of the theory because $M_{\mathrm{LS}}$ leaves $Z_{\Omega}$ covariant.

Like at the quadratic level of the action, we consider the elements $M=\left(\begin{array}{cc}A & B \\ C & D\end{array}\right) \in S p\left(\mathbb{R}^{2 D}, \omega\right)$ leaving the interaction term $\int \mathrm{d}^{4} x \phi \star \phi \star \phi \star \phi(x)$ invariant under the action $\phi \mapsto \mu(M) \phi$. By using equations (14), we find the group generated on the one hand by the matrices $\left(\begin{array}{cc}A & 0 \\ 0 & -\Sigma\left(A^{T}\right)^{-1} \Sigma\end{array}\right)$, where $A \in S p\left(\mathbb{R}^{D}, \Sigma\right)$, which correspond to the usual symplectic group of the position space and was already known to let this term invariant; and on the other hand by the matrix $M=M_{\mathrm{LS}}$, 
defined above. The Langmann-Szabo invariance of the quartic term is indeed due to property (10).

Thus, we see that the action without harmonic term (3) respects the $S p\left(\mathbb{R}^{D}, \Sigma\right)$ symmetry of the quartic term, but not the $M_{\mathrm{LS}}$-symmetry (it is not Langmann-Szabo covariant). To include this symmetry, one can take $\mathrm{d} \mu\left(Z_{\Omega}\right)$ as the quadratic operator of the action (like in (4)). But this action breaks now the $S p\left(\mathbb{R}^{D}, \Sigma\right)$ part into $O\left(\mathbb{R}^{D}, \mathbb{1}\right) \cap S p\left(\mathbb{R}^{D}, \Sigma\right)$. The above reinterpretation is in fact a group explanation of the Langmann-Szabo duality via the metaplectic representation $\mu$.

\subsection{Superalgebraic framework}

The second mathematical interpretation of the harmonic term has been given in [11] by introducing a $\mathbb{Z}_{2}$-graded algebra and a noncommutative differential calculus based on its derivations.

Upon using basic properties of the Moyal product such as $i\left[\xi_{\mu}, f\right]_{\star}=\partial_{\mu} f$ and $\left\{\xi_{\mu}, f\right\}_{\star}=2 \xi_{\mu} f$, where we recall that $\xi_{\mu}=-\frac{1}{2} \widetilde{x}_{\mu}$, the action (4) can be reexpressed as:

$$
S(\phi)=\int \mathrm{d}^{D} x\left(\frac{1}{2}\left(i\left[\xi_{\mu}, \phi\right]_{\star}\right)^{2}+\frac{\Omega^{2}}{2}\left(\left\{\xi_{\mu}, \phi\right\}_{\star}\right)^{2}+\frac{m^{2}}{2} \phi^{2}+\lambda \phi \star \phi \star \phi \star \phi\right) .
$$

And, at the quadratic level of the action, the Langmann-Szabo duality, which exchanges $p_{\mu}$ and $\widetilde{x}_{\mu}$, can be viewed as a symmetry between commutators $i[\cdot, \cdot]_{\star}$ and anticommutators $\{\cdot, \cdot \cdot\}_{\star}$.

Moreover, by replacing the curvature $F_{\mu \nu}=\Theta_{\mu \nu}^{-1}-i\left[\mathcal{A}_{\mu}, \mathcal{A}_{\nu}\right]_{\star}^{2}$ in function of the covariant coordinate $\mathcal{A}_{\mu}$ in the gauge-invariant action (7), one finds:

$$
S(\mathcal{A})=\int \mathrm{d}^{D} x\left(\frac{1}{4}\left(i\left[\mathcal{A}_{\mu}, \mathcal{A}_{\nu}\right]_{\star}\right)^{2}+\frac{\Omega^{2}}{4}\left(\left\{\mathcal{A}_{\mu}, \mathcal{A}_{\nu}\right\}_{\star}\right)^{2}+\kappa \mathcal{A}_{\mu} \mathcal{A}_{\mu}\right)
$$

up to a constant term. For this action, the Langmann-Szabo duality does not apply but it is also symmetric under the exchange of commutators and anticommutators like in the scalar case. This symmetry, which generalizes the Langmann-Szabo duality to the gauge case, seems to be crucial for noncommutative QFT with harmonic term.

The exchange of commutators and anticommutators is very reminiscent to a grading symmetry, and this motivates the introduction of the $\mathbb{Z}_{2}$-graded associative algebra $\mathbf{A}_{\theta}^{\bullet}=\mathbf{A}_{\theta}^{0} \oplus \mathbf{A}_{\theta}^{1}$, where $\mathbf{A}_{\theta}^{0}=\mathcal{M}_{\theta}$ and $\mathbf{A}_{\theta}^{1}=\mathcal{M}_{\theta}$, with product: $\forall f=\left(f_{0}, f_{1}\right), g=\left(g_{0}, g_{1}\right) \in \mathbf{A}_{\theta}^{\bullet}$,

$$
f \cdot g=\left(f_{0} \star g_{0}+\alpha f_{1} \star g_{1}, f_{0} \star g_{1}+f_{1} \star g_{0}\right),
$$

where $\alpha$ is a real parameter. The algebra is in fact a quadratic extension of the ring $\mathcal{M}_{\theta}$, but endowed with a canonical grading compatible with the product. This algebra will be called in the following the Moyal superalgebra.

The graded center of $\mathbf{A}_{\theta}^{\bullet}$, namely the elements of $\mathbf{A}_{\theta}^{\bullet}$ which (graded-) commute with all elements, is trivial: $\mathcal{Z}^{\bullet}\left(\mathbf{A}_{\theta}\right)=\mathbb{C} \oplus 0=\mathbb{C} \mathbb{1}$, where $\mathbb{1}=(1,0)$ is the unit of the product. One can choose $f^{*}=\left(f_{0}^{\dagger}, f_{1}^{\dagger}\right)$ as involution on $\mathbf{A}_{\theta}^{\bullet}$ and $\operatorname{Tr}(f)=\int \mathrm{d}^{D} x f_{0}(x)$ as (non-graded) trace.

The graded bracket associated to $\mathbf{A}_{\theta}^{\bullet}$ is given by the following expression: $\forall f, g \in \mathbf{A}_{\theta}^{\bullet}$,

$$
[f, g]=\left(\left[f_{0}, g_{0}\right]_{\star}+\alpha\left\{f_{1}, g_{1}\right\}_{\star},\left[f_{0}, g_{1}\right]_{\star}+\left[f_{1}, g_{0}\right]_{\star}\right)
$$

in terms of the commutator and the anticommutator of $\mathcal{M}_{\theta}$, which will permit to encode the symmetry noticed above.

Then, for $\mathfrak{g}^{\bullet}$ a graded Lie subalgebra of the graded derivations ${ }^{1}$ of $\mathbf{A}_{\theta}^{\bullet}$ :

$$
\operatorname{Der}^{\bullet}\left(\mathbf{A}_{\theta}\right)=\left\{\mathfrak{X} \in \mathcal{L}\left(\mathbf{A}_{\theta}\right), \quad \forall f, g \in \mathbf{A}_{\theta}^{\bullet}, \quad \mathfrak{X}(f \cdot g)=\mathfrak{X}(f) \cdot g+(-1)^{|\mathfrak{X}||f|} f \cdot \mathfrak{X}(g)\right\},
$$

\footnotetext{
${ }^{1}$ The following definition of derivations applies rigorously only for homogeneous elements $\mathfrak{X}$ and $f$, because the degrees $|\mathfrak{X}|,|f| \in \mathbb{Z}_{2}$ are considered. Let us take as a convention that it means the sum of homogeneous components thanks to the linearity.
} 
one can construct the derivation-based differential calculus $\Omega^{\bullet \bullet \bullet}\left(\mathbf{A}_{\theta} \mid \mathfrak{g}\right)$ restricted to the derivations involved in $\mathfrak{g}^{\bullet}$. A $n$-form $\omega \in \Omega^{n, \bullet}\left(\mathbf{A}_{\theta} \mid \mathfrak{g}\right)$ is a n-linear map $\omega:\left(\mathfrak{g}^{\bullet}\right)^{n} \rightarrow \mathbf{A}_{\theta}^{\bullet}$ with the property of graded antisymmetry: $\forall \mathfrak{X}_{j} \in \mathfrak{g}^{\bullet}$,

$$
\omega\left(\mathfrak{X}_{1}, \ldots, \mathfrak{X}_{i}, \mathfrak{X}_{i+1}, \ldots, \mathfrak{X}_{n}\right)=-(-1)^{\left|\mathfrak{X}_{i}\right|\left|\mathfrak{X}_{i+1}\right|} \omega\left(\mathfrak{X}_{1}, \ldots, \mathfrak{X}_{i+1}, \mathfrak{X}_{i}, \ldots, \mathfrak{X}_{n}\right) .
$$

For more details on the construction and properties of this noncommutative differential calculus, see $[56,57]$ in the case of associative algebras, and [11] for graded associative algebras.

Let us now choose the graded Lie subalgebra $\mathfrak{g}^{\bullet}$ of derivations. We introduce $\tilde{\mathfrak{g}}^{\bullet}$ as generated by the following elements $(0, i),\left(i \xi_{\mu}, 0\right),\left(0, i \xi_{\mu}\right)$ and $\left(i \eta_{\mu \nu}, 0\right)$, where $\eta_{\mu \nu}=2 \xi_{\mu} \xi_{\nu}=\frac{1}{2} \widetilde{x}_{\mu} \widetilde{x}_{\nu}$, which satisfy the relations ${ }^{2}$ :

$$
\begin{aligned}
& {[(0, i),(0, i)]=-2 \alpha \mathbb{1}, \quad\left[\left(0, i \xi_{\mu}\right),(0, i)\right]=\left(-2 \alpha \xi_{\mu}, 0\right), \quad\left[\left(i \xi_{\mu}, 0\right),\left(i \xi_{\nu}, 0\right)\right]=i \Theta_{\mu \nu}^{-1} \mathbb{1},} \\
& {\left[\left(i \xi_{\mu}, 0\right),\left(0, i \xi_{\nu}\right)\right]=\left(0, i \Theta_{\mu \nu}^{-1}\right), \quad\left[\left(0, i \xi_{\mu}\right),\left(0, i \xi_{\nu}\right)\right]=\left(-\alpha \eta_{\mu \nu}, 0\right),} \\
& {\left[\left(i \eta_{\mu \nu}, 0\right),\left(i \xi_{\rho}, 0\right)\right]=\left(\frac{i}{2} \xi_{\mu} \Theta_{\nu \rho}^{-1}+\frac{i}{2} \xi_{\nu} \Theta_{\mu \rho}^{-1}, 0\right),} \\
& {\left[\left(i \eta_{\mu \nu}, 0\right),\left(0, i \xi_{\rho}\right)\right]=\left(0, \frac{i}{2} \xi_{\mu} \Theta_{\nu \rho}^{-1}+\frac{i}{2} \xi_{\nu} \Theta_{\mu \rho}^{-1}\right),} \\
& {\left[\left(i \eta_{\mu \nu}, 0\right),\left(i \eta_{\rho \sigma}, 0\right)\right]=\left(\frac{i}{2} \eta_{\mu \rho} \Theta_{\nu \sigma}^{-1}+\frac{i}{2} \eta_{\mu \sigma} \Theta_{\nu \rho}^{-1}+\frac{i}{2} \eta_{\nu \rho} \Theta_{\mu \sigma}^{-1}+\frac{i}{2} \eta_{\nu \sigma} \Theta_{\mu \rho}^{-1}, 0\right) .}
\end{aligned}
$$

Then, we take $\mathfrak{g}^{\bullet}=\operatorname{ad} \tilde{\mathfrak{g}}^{\bullet}$. Since the (graded) adjoint representation evaluated on $f \in \mathbf{A}_{\theta}^{\bullet}$ is a graded derivation: $\operatorname{ad}_{f} g=[f, g]$, and because of relations $(15), \mathfrak{g}^{\bullet}$ is a graded Lie algebra.

The differential acts on the elements of $\mathbf{A}_{\theta}^{\bullet}$ as:

$$
\begin{aligned}
& \mathrm{d} f\left(\operatorname{ad}_{\left(i \xi_{\mu}, 0\right)}\right)=\left(\partial_{\mu} f_{0}, \partial_{\mu} f_{1}\right), \quad \mathrm{d} f\left(\operatorname{ad}_{\left(0, i \xi_{\mu}\right)}\right)=\left(i \alpha \widetilde{x}_{\mu} f_{1}, \partial_{\mu} f_{0}\right), \\
& \mathrm{d} f\left(\operatorname{ad}_{(0, i)}\right)=\left(-2 i f_{1}, 0\right), \quad \mathrm{d} f\left(\operatorname{ad}_{\left(i \eta_{\mu \nu}, 0\right)}\right)=-\frac{1}{4}\left(\widetilde{x}_{\mu} \partial_{\nu} f_{0}+\widetilde{x}_{\nu} \partial_{\mu} f_{0}, \widetilde{x}_{\mu} \partial_{\nu} f_{1}+\widetilde{x}_{\nu} \partial_{\mu} f_{1}\right) .
\end{aligned}
$$

Because of the $\mathbb{Z}_{2}$-grading, the space $\Omega^{\bullet \bullet \bullet}\left(\mathbf{A}_{\theta} \mid \mathfrak{g}\right)$ is infinite-dimensional, and one defines neither a Hodge operation for noncommutative metrics in this case, nor the related notion of Laplacian. However, it is still possible to define a scalar action,

$$
S=\operatorname{Tr}\left(\sum_{a}\left|\operatorname{ad}_{a}(\phi, \phi)\right|^{2}\right),
$$

mimicking the ordinary case. In (16), we have imposed that $\phi_{0}=\phi_{1}=\phi \in \mathcal{M}_{\theta}$ and the sum on $a$ is over $\left\{\left(0, \frac{i}{\sqrt{\theta}}\right),\left(i \xi_{\mu}, 0\right),\left(0, i \xi_{\mu}\right),\left(i \sqrt{\theta} \eta_{\mu \nu}, 0\right)\right\}$, where $\sqrt{\theta}$ appears because of dimensional reasons. Then, we find

$$
S(\phi)=\int d^{D} x\left((1+2 \alpha)\left(\partial_{\mu} \phi\right)^{2}+\alpha^{2}\left(\widetilde{x}_{\mu} \phi\right)^{2}+\frac{4 \alpha^{2}}{\theta} \phi^{2}\right)
$$

as a part of the action (16), so that the Grosse-Wulkenhaar model (4) emerges from this superalgebraic framework. Moreover, the Langmann-Szabo duality is related to the grading symmetry $\left(i \xi_{\mu}, 0\right) \rightleftarrows\left(0, i \xi_{\mu}\right)$ at the quadratic level of the action (4) (see Subsection 4.1).

In this setting, a graded connection on $\mathbf{A}_{\theta}^{\bullet}$ (considered as a right module on itself) is a homogeneous linear map of degree $0, \nabla: \mathbf{A}_{\theta}^{\bullet} \rightarrow \Omega^{1, \bullet}\left(\mathbf{A}_{\theta} \mid \mathfrak{g}\right)$ such that: $\forall \mathfrak{X} \in \mathfrak{g}, \forall f, g \in \mathbf{A}_{\theta}^{\bullet}$,

$$
\nabla(f \cdot g)(\mathfrak{X})=f \cdot \mathrm{d} g(\mathfrak{X})+(-1)^{|g||\mathfrak{X}|} \nabla(f)(\mathfrak{X}) \cdot g .
$$

\footnotetext{
${ }^{2}$ The missing relations vanish.
} 
Let us denote by $A_{\mu}^{0}, A_{\mu}^{1}, \varphi$ and $G_{\mu \nu}$ the gauge potentials associated to $\nabla$ :

$$
\begin{array}{ll}
\nabla(\mathbb{1})\left(\operatorname{ad}_{\left(i \xi_{\mu}, 0\right)}\right)=\left(-i A_{\mu}^{0}, 0\right), & \nabla(\mathbb{1})\left(\operatorname{ad}_{\left(0, i \xi_{\mu}\right)}\right)=\left(0,-i A_{\mu}^{1}\right), \\
\nabla(\mathbb{1})\left(\operatorname{ad}_{(0, i)}\right)=(0,-i \varphi), & \nabla(\mathbb{1})\left(\operatorname{ad}_{\left(i \eta_{\mu \nu}, 0\right)}\right)=\left(-i G_{\mu \nu}, 0\right) .
\end{array}
$$

The gauge transformations are then dictated by the theory. They are determined by unitary elements $g$ of $\mathcal{M}_{\theta}\left(g^{\dagger} \star g=g \star g^{\dagger}=1\right)$ acting on the fields as:

$$
\begin{aligned}
& \left(A_{\mu}^{0}\right)^{g}=g \star A_{\mu}^{0} \star g^{\dagger}+i g \star \partial_{\mu} g^{\dagger}, \quad\left(A_{\mu}^{1}\right)^{g}=g \star A_{\mu}^{1} \star g^{\dagger}+i g \star \partial_{\mu} g^{\dagger}, \\
& \varphi^{g}=g \star \varphi \star g^{\dagger}, \quad\left(G_{\mu \nu}\right)^{g}=g \star G_{\mu \nu} \star g^{\dagger}-\frac{i}{4} g \star\left(\widetilde{x}_{\mu} \partial_{\nu} g^{\dagger}\right)-\frac{i}{4} g \star\left(\widetilde{x}_{\nu} \partial_{\mu} g^{\dagger}\right) .
\end{aligned}
$$

Then, the graded curvature $F_{\mathfrak{X}, \mathfrak{Y}}$, for $\mathfrak{X}, \mathfrak{Y} \in \mathfrak{g}^{\bullet}$, can be computed:

$$
F_{\mathfrak{X}, \mathfrak{Y}}=\mathfrak{X}\left(A_{\mathfrak{Y}}\right)-(-1)^{|\mathfrak{X}|,|\mathfrak{Y}|} \mathfrak{Y}\left(A_{\mathfrak{X}}\right)-i\left[A_{\mathfrak{X}}, A_{\mathfrak{Y}}\right]-A_{[\mathfrak{X}, \mathfrak{Y}]}
$$

in term of the potentials $A_{\mathfrak{X}}=i \nabla(\mathbb{1})(\mathfrak{X})$, and it turns out that the Yang-Mills action ${ }^{3}$ $\operatorname{Tr}\left(\left|F_{\mathrm{ad}_{a}, \mathrm{ad}_{b}}\right|^{2}\right)$ for this model takes the following form ${ }^{4}$ :

$$
\begin{aligned}
S(A, \varphi, G)= & \int d^{D} x\left((1+2 \alpha) F_{\mu \nu} \star F_{\mu \nu}+\alpha^{2}\left\{\mathcal{A}_{\mu}, \mathcal{A}_{\nu}\right\}_{\star}^{2}\right. \\
& +\frac{8}{\theta}\left(2(D+1)(1+\alpha)+\alpha^{2}\right) \mathcal{A}_{\mu} \star \mathcal{A}_{\mu}+2 \alpha\left(\partial_{\mu} \varphi-i\left[A_{\mu}, \varphi\right]_{\star}\right)^{2} \\
& +2 \alpha^{2}\left(\widetilde{x}_{\mu} \varphi+\left\{A_{\mu}, \varphi\right\}_{\star}\right)^{2}-4 \alpha \sqrt{\theta} \varphi \Theta_{\mu \nu}^{-1} F_{\mu \nu}+\frac{2 \alpha(D+2 \alpha)}{\theta} \varphi^{2}-\frac{8 \alpha^{2}}{\sqrt{\theta}} \varphi \star \varphi \star \varphi \\
& +4 \alpha^{2} \varphi \star \varphi \star \varphi \star \varphi-\alpha\left[\mathcal{G}_{\mu \nu}, \varphi\right]_{\star}^{2}+2 \alpha^{2}\left\{\mathcal{A}_{\mu}, \mathcal{A}_{\nu}\right\}_{\star} \mathcal{G}_{\mu \nu} \\
& -8 i(1+\alpha) \Theta_{\nu \mu}^{-1}\left[\mathcal{A}_{\rho}, \mathcal{A}_{\mu}\right]_{\star} \star \mathcal{G}_{\nu \rho}+8 i \mathcal{G}_{\mu \nu} \star \mathcal{G}_{\rho \sigma} \star\left(\Theta_{\mu \rho}^{-1} \mathcal{G}_{\nu \sigma}+\Theta_{\nu \sigma}^{-1} \mathcal{G}_{\mu \rho}+\Theta_{\nu \rho}^{-1} \mathcal{G}_{\mu \sigma}\right. \\
& \left.+\Theta_{\mu \sigma}^{-1} \mathcal{G}_{\nu \rho}\right)+\left(\frac{16}{\theta^{2}}(D+2) \alpha^{2}\right) \mathcal{G}_{\mu \nu} \star \mathcal{G}_{\mu \nu}+2 \mathcal{G}_{\mu \nu} \star \mathcal{G}_{\mu \nu} \star \mathcal{G}_{\rho \sigma} \star \mathcal{G}_{\rho \sigma} \\
& \left.-2 \mathcal{G}_{\mu \nu} \star \mathcal{G}_{\rho \sigma} \star \mathcal{G}_{\mu \nu} \star \mathcal{G}_{\rho \sigma}\right),
\end{aligned}
$$

where $\mathcal{A}_{\mu}=A_{\mu}+\frac{1}{2} \widetilde{x}_{\mu}$ and $\mathcal{G}_{\mu \nu}=G_{\mu \nu}-\frac{1}{2} \widetilde{x}_{\mu} \widetilde{x}_{\nu}$.

The pure part of the action (17) in the variable $A_{\mu}$ (or $\mathcal{A}_{\mu}$ ) gives the action (7), while the pure part in the variable $\varphi$ is a scalar action, with $\star$-polynomial and harmonic term, of the type (4). The coupling between the two fields is the standard one [45] for a gauge field $A_{\mu}$ and a scalar field $\varphi$ in the "adjoint representation" of the gauge group, but there is also an additional BFterm $\left(-4 \alpha \sqrt{\theta} \varphi \Theta_{\mu \nu}^{-1} F_{\mu \nu}\right)$. Therefore, both Grosse-Wulkenhaar model and its associated gauge theory can be obtained in this graded formalism by computing a graded curvature for the noncommutative derivation-based differential calculus of the Moyal superalgebra $\mathbf{A}_{\theta}^{\bullet}$. For more details on the calculations, see [11]. Notice that in the different context of commutative theories, the quadratic operator with harmonic term has been obtained in [58] by considering also a $\mathbb{Z}_{2^{-}}$ graded Lie algebra of operators acting on a certain Hilbert space.

We will see in Subsection 4.1 that this approach leading to the action (17) reproduces the Langmann-Szabo duality by a grading symmetry, and it is well adapted to gauge theory, contrary to the Langmann-Szabo duality itself. However, this theory involves two supplementary fields: one $(\varphi)$ can be interpreted as a Higgs field ${ }^{5}$, but the interpretation of the other one $\left(G_{\mu \nu}\right)$ is less obvious. In [11], it has been suggested that it could play the role of the symmetric counterpart of a BF-field.

\footnotetext{
${ }^{3}$ With an implicit summation on $a, b$ like in the scalar case (16) above.

${ }^{4}$ By identifying the fields $A_{\mu}^{0}=A_{\mu}^{1}=A_{\mu}$, which have the same gauge transformations.

${ }^{5}$ Note that such a Higgs field has already been obtained by a similar (but non-graded) procedure on the Moyal space in [59].
} 


\subsection{Noncommutative scalar curvature}

In this subsection, we will describe how to obtain the harmonic term $x^{2}$ of the action (4) as a noncommutative scalar curvature [12]. Indeed, the Moyal algebra can be approximated by matrix algebras, just as the Heisenberg algebra is a limit of "truncated Heisenberg algebras". These are (non-graded) quadratic algebras, on which a noncommutative differential calculus has been constructed in [12] by using the Cartan frame formalism [60]. Then, linear connections can be introduced and scalar curvature of metric connections can be computed [61]. By taking the limit of these curvatures, one finds the term $x^{2}$. We expose here the $D=2$-dimensional case for simplicity reasons.

It has been shown in [14] that the eigenfunctions $\left(f_{m n}(x)\right)$, for $m, n, \in \mathbb{N}$ in the two-dimensional case, of the harmonic oscillator $H=\frac{x^{2}}{2}$ provide a basis of $\mathcal{M}_{\theta}$ (with respect to its Fréchet structure), called the matrix basis. These functions are defined by:

$$
H \star f_{m n}=\theta\left(m+\frac{1}{2}\right) f_{m n}, \quad f_{m n} \star H=\theta\left(n+\frac{1}{2}\right) f_{m n},
$$

and they are Schwartz functions. It turns out that they satisfy the following properties:

$$
f_{m n} \star f_{k l}=\delta_{n k} f_{m l}, \quad \int \mathrm{d}^{2} x f_{m n}(x)=2 \pi \theta \delta_{m n}, \quad f_{m n}^{\dagger}=f_{n m} .
$$

Each element $g \in \mathcal{M}_{\theta}$ can then be written as:

$$
g=\sum_{m, n \in \mathbb{N}} g_{m n} f_{m n}
$$

where the coefficients $g_{m n} \in \mathbb{C}$ are given by $g_{m n}=\frac{1}{2 \pi \theta} \int \mathrm{d}^{2} x\left(g f_{n m}\right)$. Because of properties (18), the coefficients $\left(g_{m n}\right)$ behave like matrices under the product, the trace and the involution. For example, the coefficient of the Moyal product of two elements is just the matrix product of the coefficients of these two elements: $(g \star h)_{m n}=\sum_{k \in \mathbb{N}} g_{m k} h_{k n}$. In this identification, $\mathcal{M}_{\theta}$ is a limit of finite-dimensional matrix algebras.

Let us exhibit what could be an approximation of the Heisenberg algebra $\mathfrak{h}_{1}=\left\langle 1, x_{1}, x_{2}\right\rangle$ (satisfying $\left[x_{1}, x_{2}\right]_{\star}=-i \theta$ ) in this context. The matrix coefficient of the function $x \mapsto 1$ is $\delta_{m n}$, while those of the coordinates are:

$$
\left(x_{1}\right)_{m n}=\sqrt{\frac{\theta}{2}}\left(\sqrt{n} \delta_{m+1, n}+\sqrt{m} \delta_{m, n+1}\right), \quad\left(x_{2}\right)_{m n}=i \sqrt{\frac{\theta}{2}}\left(\sqrt{n} \delta_{m+1, n}-\sqrt{m} \delta_{m, n+1}\right) .
$$

Then, following [12], we introduce the $n \times n$ matrices:

$$
\begin{gathered}
X_{n}=\frac{1}{\sqrt{2}}\left(\begin{array}{ccccc}
0 & 1 & 0 & & \\
1 & 0 & \sqrt{2} & & \\
0 & \sqrt{2} & 0 & \ddots & \\
& & \ddots & & \sqrt{n-1} \\
& & & \sqrt{n-1} & 0
\end{array}\right), \\
Y_{n}=\frac{i}{\sqrt{2}}\left(\begin{array}{ccccc}
0 & -1 & 0 & & \\
1 & 0 & -\sqrt{2} & & \\
0 & \sqrt{2} & 0 & \ddots & \\
& & \ddots & & -\sqrt{n-1}
\end{array}\right),
\end{gathered}
$$


which verify the commutation relation $\left[X_{n}, Y_{n}\right]=i\left(\mathbb{1}-Z_{n}\right)$, where $\mathbb{1}$ is the unit matrix and $Z_{n}=\left(\begin{array}{cccc}0 & 0 & & \\ 0 & 0 & \ddots & \\ & \ddots & & 0 \\ & & 0 & n\end{array}\right)$. By considering these three generators, one obtains the following quadratic algebra, called the "truncated Heisenberg algebra" $\mathfrak{h}_{1}^{(n)}$ :

$$
\left[X_{n}, Y_{n}\right]=i\left(1-Z_{n}\right), \quad\left[X_{n}, Z_{n}\right]=i\left(Y_{n} Z_{n}+Z_{n} Y_{n}\right), \quad\left[Y_{n}, Z_{n}\right]=-i\left(X_{n} Z_{n}+Z_{n} X_{n}\right) .
$$

When $n \rightarrow \infty$, one has: $X_{n} \rightarrow \frac{1}{\sqrt{\theta}} x_{1}, Y_{n} \rightarrow-\frac{1}{\sqrt{\theta}} x_{2}$ and $Z_{n} \rightarrow 0$, so that these quadratic algebras $\mathfrak{h}_{1}^{(n)}$ approximate the Heisenberg algebra $\mathfrak{h}_{1}$, up to a rescaling of the generators.

The momenta associated to these coordinates are ${ }^{6}: p_{1}=i Y, p_{2}=-i X$ and $p_{3}=i\left(Z-\frac{1}{2}\right)$, satisfying the quadratic relations:

$$
\left[p_{1}, p_{2}\right]=p_{3}-\frac{i}{2}, \quad\left[p_{2}, p_{3}\right]=p_{1}-i\left(p_{1} p_{3}+p_{3} p_{1}\right), \quad\left[p_{3}, p_{1}\right]=p_{2}-i\left(p_{2} p_{3}+p_{3} p_{2}\right) .
$$

Note that the quadratic algebra of [12] contains moreover normalization factors $\epsilon$ and $\mu$ which we do not write here for simpler notations. If we apply the Cartan frame formalism [60] to this quadratic algebra, we obtain a noncommutative differential calculus, whose one-forms are generated by the frame $\left\{\theta^{i}\right\}$, where $\theta^{i}: \operatorname{Int}\left(\mathfrak{h}_{1}^{(n)}\right) \rightarrow \mathbb{C}$ satisfies $\theta^{i}\left(\operatorname{ad}_{p_{j}}\right)=\delta_{j}^{i}$. On one-forms, the product and the differential are defined by:

$$
\begin{aligned}
& \left(\theta^{i}\right)^{2}=0, \quad \theta^{1} \theta^{2}=-\theta^{2} \theta^{1}, \quad \theta^{2} \theta^{3}=-i \theta^{1} \theta^{3}, \quad \theta^{3} \theta^{2}=i \theta^{3} \theta^{1}, \\
& \mathrm{~d} \theta^{1}=-\left(p_{3}-\frac{i}{2}\right)\left(\theta^{1} \theta^{3}+\theta^{3} \theta^{1}\right), \quad \mathrm{d} \theta^{2}=i\left(p_{3}-\frac{i}{2}\right)\left(\theta^{1} \theta^{3}-\theta^{3} \theta^{1}\right), \\
& \mathrm{d} \theta^{3}=-\theta^{1} \theta^{2}-p_{1}\left(\theta^{1} \theta^{3}+\theta^{3} \theta^{1}\right)+i p_{2}\left(\theta^{1} \theta^{3}-\theta^{3} \theta^{1}\right) .
\end{aligned}
$$

In this setting, one denotes $\omega^{i}{ }_{j}=\omega^{i}{ }_{k j} \theta^{k}$ the one-form associated to a linear connection. In [12] is given an approximated solution (in the commutative limit) of a torsion-free linear connection compatible with the (noncommutative) metric $g_{i j}=\delta_{i j}$ :

$$
\begin{aligned}
& \omega^{1}{ }_{2}=-\omega^{2}{ }_{1}=\left(-\frac{1}{2}+2 i p_{3}\right) \theta^{3}, \quad \omega^{1}{ }_{3}=-\omega^{3}{ }_{1}=\frac{1}{2} \theta^{2}+2 i p_{2} \theta^{3}, \\
& \omega^{2}{ }_{3}=-\omega^{3}{ }_{2}=-\frac{1}{2} \theta^{1}-2 i p_{1} \theta^{3} .
\end{aligned}
$$

Then, one can obtain the noncommutative Riemann curvature of this connection:

$$
\Omega^{i}{ }_{j}=\mathrm{d} \omega^{i}{ }_{j}+\omega^{i}{ }_{k} \omega^{k}{ }_{j}=\frac{1}{2} R_{j k l}^{i} \theta^{k} \theta^{l},
$$

and its scalar curvature $R=g^{i j} R_{i k j}^{k}$. In this case, one finds [12]:

$$
R=\frac{11}{2}+4 i p_{3}+8\left(p_{1}^{2}+p_{2}^{2}\right)=\frac{15}{2}-4 Z-8\left(X^{2}+Y^{2}\right)
$$

In the limit $n \rightarrow \infty$, the scalar curvature takes the form: $R=\frac{15}{2 \theta}-8 \widetilde{x}^{2}$ up to a rescaling.

On a commutative but curved manifold, the classical action of a scalar theory involves naturally a term like $\int \sqrt{g} \mathrm{~d} x\left(\xi R \phi^{2}\right)$, where $R$ is the scalar curvature of the (pseudo-) Riemannian

\footnotetext{
${ }^{6}$ We omit the subscript $n$.
} 
manifold (see [62] and references therein). This term is indeed automatically generated by quantum corrections, so that the parameter $\xi$ has to be renormalized.

In that situation, the harmonic term $\widetilde{x}^{2}$ can be interpreted as a noncommutative scalar curvature, and the Grosse-Wulkenhaar model (4) would be a generalization of curved scalar field theories to a noncommutative framework. Notice that this harmonic tem is not generated by quantum corrections in the scalar model (3), contrary to the curved commutative models.

However, this interpretation is not directly possible on the Moyal algebra. Indeed, the Heisenberg algebra $\mathfrak{h}_{1}$ is a Lie algebra and not a quadratic algebra. Therefore, the application of the Cartan formalism would give in this case a vanishing scalar curvature $R$. One had to approximate this algebra by the "truncated Heisenberg algebras".

Recently, the gauge model associated to this noncommutative differential calculus has been exhibited in [13]. As usual, the curvature $\mathrm{F}$, or field strength, can be computed in terms of the gauge potential $A$ associated to a gauge connection by the formula: $\mathrm{F}=\mathrm{d} A+A^{2}$, where $A=A_{i} \theta^{i}$ and $\mathrm{F}=\frac{1}{2} \mathrm{~F}_{i j} \theta^{i} \theta^{j}$ are respectively a one-form and a two-form. One obtains:

$$
\begin{aligned}
& \mathrm{F}_{12}=\left[p_{1}, A_{2}\right]-\left[p_{2}, A_{1}\right]+\left[A_{1}, A_{2}\right]-A_{3}, \\
& \mathrm{~F}_{13}=\left[p_{1}+A_{1}, A_{3}\right]-i\left\{p_{2}+A_{2}, A_{3}\right\}+2 A_{2} Z, \\
& \mathrm{~F}_{23}=\left[p_{2}+A_{2}, A_{3}\right]+i\left\{p_{1}+A_{1}, A_{3}\right\}-2 A_{1} Z .
\end{aligned}
$$

Then, we restrict to the case of the Moyal space: $n \rightarrow \infty$ (which means $Z \rightarrow 0$ ). By considering the gauge transformations of the potential:

$$
A_{1} \mapsto g A_{1} g^{-1}+g\left[p_{1}, g^{-1}\right], \quad A_{2} \mapsto g A_{2} g^{-1}+g\left[p_{2}, g^{-1}\right], \quad A_{3} \mapsto g A_{3} g^{-1},
$$

we denote $A_{3}=\varphi$, considered as a scalar field in the adjoint representation. The curvature takes the form:

$$
\mathrm{F}_{12}=\left[\mathcal{A}_{1}, \mathcal{A}_{2}\right]_{\star}+i-\varphi, \quad \mathrm{F}_{13}=\left[\mathcal{A}_{1}, \varphi\right]_{\star}-i\left\{\mathcal{A}_{2}, \varphi\right\}_{\star}, \quad \mathrm{F}_{23}=\left[\mathcal{A}_{2}, \varphi\right]_{\star}+i\left\{\mathcal{A}_{1}, \varphi\right\}_{\star},
$$

where $\mathcal{A}_{\mu}$ is the covariant coordinate introduced in Subsection 3.2. By using a Hodge operation defined in [13], the following Yang-Mills action is provided ${ }^{7}$ :

$$
\begin{aligned}
S(A, \varphi)= & \int \mathrm{d}^{2} x\left(\frac{\left(1-\epsilon^{2}\right)}{4} F_{\mu \nu} \star F_{\mu \nu}+\frac{\left(5-\epsilon^{2}\right) \mu^{2}}{2} \varphi^{2}+\frac{\mu \theta\left(1-\epsilon^{2}\right)}{2} \varphi \Theta_{\mu \nu}^{-1} F_{\mu \nu}\right. \\
& \left.-i \epsilon \theta(\varphi \star \varphi) \Theta_{\mu \nu}^{-1} F_{\mu \nu}-\frac{1}{2}\left(\partial_{\mu} \varphi-i\left[A_{\mu}, \varphi\right]_{\star}\right)^{2}-\frac{\epsilon^{2}}{2}\left(\widetilde{x}_{\mu} \varphi+\left\{A_{\mu}, \varphi\right\}_{\star}\right)^{2}\right),
\end{aligned}
$$

where $F_{\mu \nu}=\partial_{\mu} A_{\nu}-\partial_{\nu} A_{\mu}-i\left[A_{\mu}, A_{\nu}\right]_{\star}$ is the standard curvature for the Moyal algebra, and $\mu, \nu \in\{1,2\}$.

This action is completed by the following gauge fixing term: $S_{\text {gf }}=\int s\left(\bar{c} \mathcal{F}+\frac{\alpha}{2} \bar{c} B\right)$, where $\mathcal{F}$ is the gauge, $c$ the ghost field, $\bar{c}$ the antighost field, $B$ the auxiliary field, and the Slavnov operator $s$ is acting as:

$$
s A_{\mu}=\partial_{\mu} c-i\left[A_{\mu}, c\right]_{\star}, \quad s \varphi=-i[\varphi, c]_{\star}, \quad s c=-c^{2}, \quad s \bar{c}=B, \quad s B=0 .
$$

Here, the chosen gauge is the covariant one:

$$
\mathcal{F}=\nabla_{i} A_{i}=\left[p_{i}, A_{i}\right]+A_{j} \omega^{i}{ }_{i j}=\partial_{\nu} A_{\nu}-2 \mu^{2} x_{\nu} A_{\nu},
$$

where $\omega($ and $\nabla)$ is the linear connection defined in the first part of this subsection.

\footnotetext{
${ }^{7}$ This is a reformulation of what has been found in [13] in our notations. We also have reintroduced the parameters $\epsilon$ and $\mu$.
} 
One can observe that the pure part of the action (19) in the variable $A_{\mu}$ is of the type (6), while the other terms describe a scalar field with a harmonic term, coupled to $A_{\mu}$, and with BF-terms. Except the special term $\int(\varphi \star \varphi) \Theta_{\mu \nu}^{-1} F_{\mu \nu}$, all the terms of (19) are included in (17). However, the term $\int\left\{\mathcal{A}_{\mu}, \mathcal{A}_{\nu}\right\}_{\star}^{2}$ of (7) is missing here, so that the propagator of the gauge potential is not the Mehler kernel like in (17), but there is no problem of non-trivial vacuum more. The hope of this solution is then [13] that the UV/IR mixing of the theory be canceled by the coupling between the gauge potential and a scalar field whose propagator is the Mehler kernel.

\section{Discussion}

\subsection{The Langmann-Szabo duality as a grading exchange}

We have seen in Subsection 3.2 that a symmetry between commutators and anticommutators seems to be fundamental for both scalar theory (4) and gauge theory (7). In the scalar case, it corresponds at the quadratic level of the action to the Langmann-Szabo duality. Let us show that this duality is exactly the grading exchange. The crucial object which has led us to the group reformulation of the Langmann-Szabo duality in Subsection 3.1 was the Heisenberg algebra $\mathfrak{h}_{D}$. In this subsection, we will exhibit the link between $\mathfrak{h}_{D}$ and the Lie superalgebra of generators $\widetilde{\mathfrak{g}}^{\bullet}=\left\langle(0, i),\left(i \xi_{\mu}, 0\right),\left(0, i \xi_{\mu}\right),\left(i \eta_{\mu \nu}, 0\right)\right\rangle$ of $\mathfrak{g}^{\bullet}$ (see equation (15)).

Let $\mathfrak{h}_{D}=\mathbb{R}^{2 D} \oplus \mathbb{R}$ be the Heisenberg algebra associated to the symplectic form ${ }^{8} \omega=$ $\left(\begin{array}{cc}0 & \Sigma^{-1} \\ \Sigma^{-1} & 0\end{array}\right)$ on the phase space $\mathbb{R}^{2 D}$, and whose commutation relations are given by (12). Since we want to deform the (symplectic) position space $\left(\mathbb{R}^{D}, \Sigma\right)$, we consider an extension $\widetilde{\mathfrak{h}}_{D} \simeq \mathbb{C}^{2 D} \oplus \mathbb{C} \oplus \mathbb{C}$ of the complexification of $\mathfrak{h}_{D}: \forall x, y, p, q \in \mathbb{C}^{D}, \forall s, t, a, b \in \mathbb{C}$,

$$
[(x, p, s, a),(y, q, t, b)]=\left(0,0, \omega((x, p),(y, q)), i \theta \Sigma^{-1}(x, y)+i \alpha \theta \Sigma^{-1}(p, q)\right) .
$$

This is a very standard way to provide noncommutative position coordinates, with deformation parameter $\theta$, and noncommutative impulsion coordinates, with deformation parameter $\alpha \theta$.

Then, one can realize this extension $\widetilde{\mathfrak{h}}_{D}$ in $\mathbf{A}_{\theta}^{\bullet}$ by the following map: $(x, p, s, a) \mapsto x_{\mu} \lambda_{\mu}+$ $p_{\mu} \lambda_{\bar{\mu}}+s \lambda_{0}+a \mathbb{1}$, where we set $\lambda_{0}=(0, i \theta), \lambda_{\mu}=\left(i \theta \xi_{\mu}, 0\right)$ and $\lambda_{\bar{\mu}}=\left(0, i \theta \xi_{\mu}\right)$ in $\mathbf{A}_{\theta}^{\bullet}$. This map is an injective (non-graded) Lie algebra morphism. The non-vanishing commutation relations (with non-graded bracket) can be expressed as:

$$
\left[\lambda_{\mu}, \lambda_{\nu}\right]=i \theta \Sigma_{\mu \nu}^{-1} \mathbb{1}, \quad\left[\lambda_{\mu}, \lambda_{\bar{\nu}}\right]=\Sigma_{\mu \nu}^{-1} \lambda_{0}, \quad\left[\lambda_{\bar{\mu}}, \lambda_{\bar{\nu}}\right]=i \alpha \theta \Sigma_{\mu \nu}^{-1} \mathbb{1} .
$$

In the commutative limit $\theta \rightarrow 0$, we find indeed a Lie algebra isomorphic to $\mathfrak{h}_{D}$.

The grading of $\mathbf{A}_{\theta}^{\bullet}$ brings a natural grading on $\widetilde{\mathfrak{h}}_{D}$. The next step of this procedure can be called the "superization" of $\widetilde{\mathfrak{h}}_{D}$. Indeed, it is a (non-graded, for its bracket) Lie algebra, but now with a $\mathbb{Z}_{2}$-grading and included in a graded associative algebra. We can superize the bracket of $\widetilde{\mathfrak{h}}_{D}$ by changing only the bracket of two odd elements into their anticommutator in $\mathbf{A}_{\theta}^{\bullet}$. Then, the changed relations hold:

$$
\left[\lambda_{0}, \lambda_{0}\right]=-2 \alpha \theta^{2} \mathbb{1}, \quad\left[\lambda_{\bar{\mu}}, \lambda_{0}\right]=2 i \alpha \theta \lambda_{\mu}, \quad\left[\lambda_{\bar{\mu}}, \lambda_{\bar{\nu}}\right]=\alpha\left(\lambda_{\mu} \lambda_{\nu}+\lambda_{\nu} \lambda_{\mu}\right)=i \alpha\left(i \eta_{\mu \nu}, 0\right) .
$$

By rescaling the generators $\lambda_{i} \mapsto \frac{1}{\theta} \lambda_{i}$ and taking the closure in $\mathbf{A}_{\theta}^{\bullet}$ (under the graded bracket) of the space $\left\langle\lambda_{i}\right\rangle$, one obtains the Lie superalgebra $\widetilde{\mathfrak{g}}^{\bullet}$ described in (15).

The symplectic group $S p\left(\mathbb{R}^{2 D}, \omega\right)$ which was acting by automorphisms on $\mathfrak{h}_{D}$, is no longer a symmetry group of $\tilde{\mathfrak{g}}^{\bullet}$. Only the subgroup $S p\left(\mathbb{R}^{D}, \Sigma\right) \otimes\left(\begin{array}{ll}1 & 0 \\ 0 & 1\end{array}\right)$ survives to the above procedure.

\footnotetext{
${ }^{8}$ The inverse of $\Sigma$, instead of $\Sigma$ itself, is taken only for conventional reasons.
} 
Then, the matrix $M_{\mathrm{LS}}=\frac{\theta}{2}\left(\begin{array}{cc}0 & \frac{4}{\theta^{2}} \mathbb{1} \\ \mathbb{1} & 0\end{array}\right)$ representing the Langmann-Szabo duality at the level of the symplectic group (see Subsection 3.1) does not leave the relations (15) invariant. However, it is still an endomorphism of the graded vector space $\widetilde{\mathfrak{g}}^{\bullet}$, and it acts as:

$$
\begin{array}{ll}
M_{\mathrm{LS} \cdot}\left(i \xi_{\mu}, 0\right)=\frac{\theta}{2}\left(0, i \xi_{\mu}\right), & M_{\mathrm{LS}} \cdot\left(0, i \xi_{\mu}\right)=\frac{2}{\theta}\left(i \xi_{\mu}, 0\right), \\
M_{\mathrm{LS}} \cdot(0, i)=(0, i), & M_{\mathrm{LS}} \cdot\left(i \eta_{\mu \nu}, 0\right)=\left(i \eta_{\mu \nu}, 0\right),
\end{array}
$$

which corresponds to the grading exchange for first order polynomials (up to a rescaling) in $\mathbb{R}^{2 D}$. In Subsection 3.2, there was only a correspondence between the Langmann-Szabo duality and the grading exchange; we see here by the above procedure that both symmetries are identical. Certainly, the action of $M_{\mathrm{LS}}$ is not a symmetry of $\tilde{\mathfrak{g}}^{\bullet}$. But one restores it as a symmetry of the scalar field theory by identifying $\phi_{0}=\phi_{1}$, and of the gauge theory by identifying $A_{\mu}^{0}=A_{\mu}^{1}$ (see Subsection 3.2). Furthermore, the field $G_{\mu \nu}$ of Subsection 3.2 is auxiliary in this point of view, since it arises only from the closure of the Lie superalgebra.

Let us summarize the discussion of this subsection.

Proposition 1. In the above notations, one can obtain the Lie superalgebra $\widetilde{\mathfrak{g}}^{\bullet}$ from the Heisenberg algebra $\mathfrak{h}_{D}$ by the following three-steps procedure:

1. Extension, for the deformation quantization,

2. "Superization" in $\mathbf{A}_{\theta}^{\bullet}$,

3. Closure in $\mathbf{A}_{\theta}^{\bullet}$ under the graded bracket.

Moreover, the Langmann-Szabo duality is identical to the grading exchange $\left(i \xi_{\mu}, 0\right) \rightleftarrows\left(0, i \xi_{\mu}\right)$.

Note that an analogous procedure (but without "superization") has been considered in [10] for a rank one Hermitian symmetric space of the non-compact type, instead of $\mathbb{R}^{2 D}$.

\subsection{Relations and comparison between the different viewpoints}

We have seen in Subsection 3.1 that the Langmann-Szabo duality, which is a cyclic symplectic Fourier transformation, can explain the form of the action (4) of the Grosse-Wulkenhaar model, but it is not well adapted to its associated gauge theory. We have also observed that this duality could be reformulated in terms of the metaplectic representation of the symplectic group, constructed from the Heisenberg algebra $\mathfrak{h}_{D}$. This algebra contains indeed positions and impulsions, which are exchanged in this duality.

The superalgebraic approach, described in Subsection 3.2, is a priori a different explanation of the Grosse-Wulkenhaar model. The choice of a graded noncommutative differential calculus adapted to a certain Lie superalgebra $\tilde{\mathfrak{g}}^{\bullet}$ gives rise to the scalar action (4) so as its gauge associated theory (7). Furthermore, the analysis of Subsection 4.1 makes appear the unification of the Langmann-Szabo duality interpretation with the superalgebraic approach. Indeed, the Lie superalgebra $\widetilde{\mathfrak{g}}^{\bullet}$ can be obtained by some natural procedure from the Heisenberg algebra $\mathfrak{h}_{D}$, in the context of the graded algebra $\mathbf{A}_{\theta}^{\bullet}$. In this picture, the above noncommutative differential calculus is constructed from $\mathfrak{h}_{D}$, and the Langmann-Szabo duality is in fact the grading exchange for first order polynomials in $\mathbf{A}_{\theta}^{\bullet}:\left(i \xi_{\mu}, 0\right) \rightleftarrows\left(0, i \xi_{\mu}\right)$.

The gauge action (17), which contains (7), has now a geometrical interpretation, as constructed from a graded curvature. Moreover, because of the supplementary field $G_{\mu \nu}$, this action has the following trivial vacuum: $A_{\mu}=0, \varphi=0, G_{\mu \nu}=0$ (see [11]), contrary to the action (7). And the gauge sector of (17) (depending only on $A_{\mu}$ ) has the Mehler kernel as propagator, up to gauge fixing terms (see equation (8)), knowing that this Mehler kernel cures the UV/IR mixing in the scalar case. 
In Subsection 3.3, we have studied another viewpoint of the harmonic term $\widetilde{x}^{2}$, obtained as the limit of noncommutative scalar curvature of "truncated Heisenberg algebras" $\mathfrak{h}_{\frac{D}{2}}^{(n)}$. Indeed, the scalar curvature appears naturally in curved commutative models as generated by quantum corrections. By analogy to this situation, and even if the term $\widetilde{x}^{2}$ is not generated by quantum corrections for the action (3), one can explain this term in the action (4) to be a noncommutative scalar curvature.

The gauge model related to this interpretation has also been described in Subsection 3.3 (see equation (19)), and is quite different from (7). Since it does not contain the symmetry between commutators and anticommutators, that we have seen in Subsections 3.2 and 4.1 to be a generalization of the Langmann-Szabo duality, one concludes that this viewpoint cannot be unified with the two first ones. The hope of renormalizability of the gauge model (19) is that the coupling between the gauge field and the scalar field (with an harmonic term) would cancel the UV/IR mixing. We see that this would be a different mechanism from the gauge model (17), where the gauge sector is supposed to be already free of UV/IR mixing.

In fact, the central object of the three mathematical interpretations is the Heisenberg algebra, $\mathfrak{h}_{D}$ for the two first ones, and $\mathfrak{h}_{\frac{D}{2}}$ (and its truncations) for the last one. Of course, one may have considered the truncated algebras of $\mathfrak{h}_{D}$, and the resulting scalar curvature would have been proportional to $p^{2}+\widetilde{x}^{2}$, the operator involved in (4). However, the interpretation by analogy of the curved commutative case is then no longer valid, and the associated gauge theory is still different from (7). Therefore, there are essentially two different approaches to interpret the noncommutative quantum field theory with harmonic term, by considering $\mathfrak{h}_{D}$ or $\mathfrak{h}_{\frac{D}{2}}$, namely

the Langmann-Szabo duality and the superalgebraic approach, which are now unified, and the noncommutative scalar curvature interpretation. These both approaches provide non-equivalent gauge models, which are candidates to renormalizability.

\section{Acknowledgements}

The author thanks Pierre Bieliavsky and Jean-Christophe Wallet for interesting discussions at various stages of this work. This work was supported by the Belgian Interuniversity Attraction Pole (IAP) within the framework "Nonlinear systems, stochastic processes, and statistical mechanics" (NOSY).

\section{References}

[1] Connes A., Noncommutative geometry, Academic Press, Inc., San Diego, CA, 1994.

[2] Landi G., An introduction to noncommutative spaces and their geometries, Lecture Notes in Physics, New Series m: Monographs, Vol. 51, Springer-Verlag, Berlin, 1997.

[3] Moyal J.E., Quantum mechanics as a statistical theory, Proc. Cambridge Philos. Soc. 45 (1949), 99-124.

[4] Doplicher S., Fredenhagen K., Roberts J.E., The quantum structure of space-time at the Planck scale and quantum fields, Comm. Math. Phys. 172 (1995), 187-220, hep-th/0303037.

[5] Minwalla S., Van Raamsdonk M., Seiberg N., Noncommutative perturbative dynamics, J. High Energy Phys. 2000 (2000), no. 2, 020, 31 pages, hep-th/9912072.

[6] Gayral V., Heat-kernel approach to UV/IR mixing on isospectral deformation manifolds, Ann. Henri Poincaré 6 (2005), 991-1023, hep-th/0412233.

[7] Gayral V., Non compact isospectral deformations and quantum field theory, hep-th/0507208.

[8] Grosse H., Wulkenhaar R., Renormalisation of $\phi^{4}$ theory on noncommutative $\mathbb{R}^{4}$ in the matrix base, Comm. Math. Phys. 256 (2005), 305-374, hep-th/0401128.

[9] Langmann E., Szabo R.J., Duality in scalar field theory on noncommutative phase spaces, Phys. Lett. B 533 (2002), 168-177, hep-th/0202039. 
[10] Bieliavsky P., Gurau R., Rivasseau V., Noncommutative field theory on rank one symmetric spaces, J. Noncommut. Geom. 3 (2009), 99-123, arXiv:0806.4255.

[11] de Goursac A., Masson T., Wallet J.-C., Noncommutative $\varepsilon$-graded connections, arXiv:0811.3567.

[12] Buric M., Wohlgenannt M., Geometry of the Grosse-Wulkenhaar model, J. High Energy Phys. 2010 (2010), no. 3, 053, 17 pages, arXiv:0902.3408.

[13] Buric M., Grosse H., Madore J., Gauge fields on noncommutative geometries with curvature, arXiv:1003.2284.

[14] Gracia-Bondia J.M., Varilly J.C., Algebras of distributions suitable for phase space quantum mechanics. I, J. Math. Phys. 29 (1988), 869-879.

[15] Varilly J.C., Gracia-Bondia J.M., Algebras of distributions suitable for phase-space quantum mechanics. II. Topologies on the Moyal algebra, J. Math. Phys. 29 (1988), 880-887.

[16] Gayral V., Gracia-Bondia J.M., Iochum B., Schucker T., Varilly J.C., Moyal planes are spectral triples, Comm. Math. Phys. 246 (2004), 569-623, hep-th/0307241.

[17] Cagnache E., D'Andrea F., Martinetti P., Wallet J.-C., The spectral distance on the Moyal plane, arXiv:0912.0906.

[18] Filk T., Divergencies in a field theory on quantum space, Phys. Lett. B 376 (1996), 53-58.

[19] Magnen J., Rivasseau V., Tanasa A., Commutative limit of a renormalizable noncommutative model, Europhys. Lett. 86 (2009), 11001, 6 pages, arXiv:0807.4093.

[20] Panero M., Quantum field theory in a non-commutative space: theoretical predictions and numerical results on the fuzzy sphere, SIGMA 2 (2006), 081, 14 pages, hep-th/0609205.

[21] Panero M., Numerical simulations of a non-commutative theory: the scalar model on the fuzzy sphere, J. High Energy Phys. 2007 (2007), no. 5, 082, 20 pages, hep-th/0608202.

[22] Gurau R., Rivasseau V., Vignes-Tourneret F., Propagators for noncommutative field theories, Ann. Henri Poincaré 7 (2006), 1601-1628, hep-th/0512071.

[23] Vignes-Tourneret F., Renormalisation of non commutative field theories, PhD Thesis, math-ph/0612014.

[24] Rivasseau V., Vignes-Tourneret F., Wulkenhaar R., Renormalization of noncommutative $\phi^{4}$-theory by multiscale analysis, Comm. Math. Phys. 262 (2006), 565-594, hep-th/0501036.

[25] Gurau R., Magnen J., Rivasseau V., Vignes-Tourneret F., Renormalization of non-commutative $\Phi_{4}^{4}$ field theory in $x$ space, Comm. Math. Phys. 267 (2006), 515-542, hep-th/0512271.

[26] Gurau R., Tanasa A., Dimensional regularization and renormalization of non-commutative QFT, Ann. Henri Poincaré 9 (2008), 655-683, arXiv:0706.1147.

[27] Grosse H., Wulkenhaar R., Renormalisation of $\phi^{4}$-theory on noncommutative $\mathbb{R}^{2}$ in the matrix base, J. High Energy Phys. 2003 (2003), no. 12, 019, 26 pages, hep-th/0307017.

[28] Gurau R., Rivasseau V., Parametric representation of noncommutative field theory, Comm. Math. Phys. 272 (2007), 811-835, math-ph/0606030.

[29] Rivasseau V., Tanasa A., Parametric representation of 'critical' noncommutative QFT models, Comm. Math. Phys. 279 (2008), 355-379, math-ph/0701034.

[30] Tanasa A., Vignes-Tourneret F., Hopf algebra of non-commutative field theory, J. Noncommut. Geom. 2 (2008), 125-139, arXiv:0707.4143.

[31] Tanasa A., Kreimer D., Combinatorial Dyson-Schwinger equations in noncommutative field theory, arXiv:0907.2182.

[32] Disertori M., Rivasseau V., Two- and three-loops beta function of noncommutative $\Phi_{4}^{4}$ theory, Eur. Phys. J. C 50 (2007), 661-671, hep-th/0610224.

[33] Disertori M., Gurau R., Magnen J., Rivasseau V., Vanishing of beta function of non-commutative $\phi_{4}^{4}$ theory to all orders, Phys. Lett. B 649 (2007), 95-102, hep-th/0612251.

[34] Grosse H., Wulkenhaar R., The $\beta$-function in duality-covariant noncommutative $\phi^{4}$-theory, Eur. Phys. J. C 35 (2004), 277-282, hep-th/0402093.

[35] de Goursac A., Tanasa A., Wallet J.-C., Vacuum configurations for renormalizable non-commutative scalar models, Eur. Phys. J. C 53 (2008), 459-466, arXiv:0709.3950.

[36] de Goursac A., Noncommutative geometry, gauge theory and renormalization, PhD Thesis, arXiv:0910.5158.

[37] de Goursac A., Wallet J.-C., Symmetries of noncommutative scalar field theory, arXiv:0911.2645. 
[38] Langmann E., Szabo R.J., Zarembo K., Exact solution of quantum field theory on noncommutative phase spaces, J. High Energy Phys. 2004 (2004), no. 1, 017, 69 pages, hep-th/0308043.

[39] Vignes-Tourneret F., Renormalization of the orientable non-commutative Gross-Neveu model, Ann. Henri Poincaré 8 (2007), 427-474, math-ph/0606069.

[40] Gurau R., Magnen J., Rivasseau V., Tanasa A., A translation-invariant renormalizable non-commutative scalar model, Comm. Math. Phys. 287 (2009), 275-290, arXiv:0802.0791.

[41] Geloun J.B., Tanasa A., One-loop $\beta$ functions of a translation-invariant renormalizable noncommutative scalar model, Lett. Math. Phys. 86 (2008), 19-32, arXiv:0806.3886.

[42] Tanasa A., Translation-invariant noncommutative renormalization, SIGMA 6 (2010), 047, 13 pages, arXiv:1003.4877.

[43] Blaschke D.N., Gieres F., Kronberger E., Schweda M., Wohlgenannt M., Translation-invariant models for non-commutative gauge fields, J. Phys. A: Math. Theor. 41 (2008), 252002, 7 pages, arXiv:0804.1914.

[44] Blaschke D.N., Rofner A., Schweda M., Sedmik R.I.P., One-loop calculations for a translation invariant non-commutative gauge model, Eur. Phys. J. C 62 (2009), 433-443, arXiv:0901.1681.

[45] de Goursac A., Wallet J.-C., Wulkenhaar R., Noncommutative induced gauge theory, Eur. Phys. J. C 51 (2007), 977-987, hep-th/0703075.

[46] Wallet J.-C., Noncommutative induced gauge theories on Moyal spaces, J. Phys. Conf. Ser. 103 (2008), 012007, 20 pages, arXiv:0708.2471.

[47] Matusis A., Susskind L., Toumbas N., The IR/UV connection in the non-commutative gauge theories, J. High Energy Phys. 2000 (2000), no. 12, 002, 18 pages, hep-th/0002075.

[48] Grosse H., Wohlgenannt M., Induced gauge theory on a noncommutative space, Eur. Phys. J. C 52 (2007), 435-450, hep-th/0703169.

[49] Blaschke D.N., Grosse H., Schweda M., Non-commutative $U(1)$ gauge theory on $\mathbb{R}_{\Theta}^{4}$ with oscillator term and BRST symmetry, Europhys. Lett. 79 (2007), 61002, 3 pages, arXiv:0705.4205.

[50] Blaschke D.N., Grosse H., Kronberger E., Schweda M., Wohlgenannt M., Loop calculations for the noncommutative $U(1)$ gauge field model with oscillator term, arXiv:0912.3642.

[51] de Goursac A., On the effective action of noncommutative Yang-Mills theory, J. Phys. Conf. Ser. 103 (2008), 012010, 16 pages, arXiv:0710.1162.

[52] de Goursac A., Wallet J.-C., Wulkenhaar R., On the vacuum states for noncommutative gauge theory, Eur. Phys. J. C 56 (2008), 293-304, arXiv:0803.3035.

[53] Ilderton A., Lundin J., Marklund M., Strong field, noncommutative QED, SIGMA 6 (2010), 041, 27 pages, arXiv:1003.4184.

[54] Fischer A., Szabo R.J., UV/IR duality in noncommutative quantum field theory, arXiv:1001.3776.

[55] Folland G.B., Harmonic analysis in phase space, Annals of Mathematics Studies, Vol. 122, Princeton University Press, Princeton, NJ, 1989.

[56] Dubois-Violette M., Dérivations et calcul différentiel non commutatif, C.R. Acad. Sci. Paris Sér. I Math. 307 (1988), 403-408.

[57] Masson T., Examples of derivation-based differential calculi related to noncommutative gauge theories, Int. J. Geom. Methods Mod. Phys. 5 (2008), 1315-1336, arXiv:0810.4815.

[58] Wulkenhaar R., Non-compact spectral triples with finite volume, arXiv:0907.1351.

[59] Cagnache E., Masson T., Wallet J.-C., Noncommutative Yang-Mills-Higgs actions from derivation-based differential calculus, J. Noncommut. Geom., to appear, arXiv:0804.3061.

[60] Madore J., Mourad J., Quantum space-time and classical gravity, J. Math. Phys. 39 (1998), 423-442, gr-qc/9607060.

[61] Dubois-Violette M., Madore J., Masson T., Mourad J., On curvature in noncommutative geometry, J. Math. Phys. 37 (1996), 4089-4102, q-alg/9512004.

[62] Hollands S., Wald R.M., On the renormalization group in curved spacetime, Comm. Math. Phys. 237 (2003), 123-160, gr-qc/0209029. 